\title{
The Affordable Care Act: Disparities in emergency department use for mental health diagnoses in young adults
}

\author{
Justin Yanuck ${ }^{1}$, Bryson Hicks ${ }^{1}$, Craig Anderson ${ }^{1}$, John Billimek ${ }^{2}$, Shahram Lotfipour ${ }^{1}$, Bharath Chakravarthy ${ }^{1}$ \\ ${ }^{1}$ Division of Emergency Medicine, University of California, Irvine, Orange, California 92868, USA \\ ${ }^{2}$ Division of General Internal Medicine and Department of Family Medicine, Irvine School of Medicine, Irvine, Orange, \\ California 92868, USA
}

Corresponding Author: Justin Yanuck, Email: jyanuck@uci.edu

BACKGROUND: There is little consensus as to the effects of insurance expansion on emergency department (ED) utilization for mental health purposes. We aimed to study the race specific association between the dependent coverage provision of the Affordable Care Act (ACA) and changes in young adults' usage of emergency department services for psychiatric diagnoses.

METHODS: We utilized a Quasi-Experimental analysis of ED use in California from 2009-2011 for behavioral health diagnoses of individuals aged 19 to 31 years. Analysis used a difference-indifferences approach comparing those targeted by the ACA dependent provision (19-25 years) and those who were not (27 to 31 years), evaluating changes in ED visit rates per 1000 in California. Primary outcomes measured included the quarterly ED visit rates with any psychiatric diagnosis. Subgroups were analyzed for differences based on race and gender.

RESULTS: The ACA dependent provision was associated with 0.05 per 1000 people fewer psychiatric ED visits among the treatment group (19-25 years) compared to the control group (27-31 years). Hispanics and Asian/Pacific Islanders were the only racial subgroups who did not see this significant reduction and were the only racial subgroups that did not see significant gains in the proportion of psychiatric ED visits covered by private insurance.

CONCLUSION: The ACA dependent provision was associated with a modest reduction in the growth rate of ED use for psychiatric reasons, however, racial disparities in the effect of this provision exist for patients of Hispanic and Asian/Pacific Islander racial groups.

KEY WORDS: Affordable Care Act; Mental health; Disparities; Health Policy

World J Emerg Med 2017;8(3):206-213

DOI: 10.5847/wjem.j.1920-8642.2017.03.008

\section{INTRODUCTION}

In 2010 there were roughly 51 million uninsured adults in the United States, and while lack of health insurance limits access to many aspects of health care, emergency departments (EDs) are mandated to provide care to all patients regardless of insurance status. ${ }^{[1-3]}$ In the United States, use of EDs has steadily increased. From 1991 to 2011, the number of annual visits to EDs rose $46 \%$, and in 2011 ED costs accounted for $2 \%-10 \%$ of the total cost of US health care. ${ }^{[4,5]}$ In $2006,20 \%$ of adult ED visits carried a primary psychiatric diagnosis (approx 4.7 million visits per year). ${ }^{[6]}$ While the range of presenting complaints to the ED is vast, psychiatric related $\mathrm{ED}$ visits represent a large component of ED users.

On March 23, 2010, the Affordable Care Act (ACA) was enacted, which contained provisions that: expanded 
health insurance coverage for patients with mental health care needs through Medicaid expansions, allowed young adults aged 19 to 25 to remain as dependents on their parents' plans, and established health insurance exchanges for cheaper and more expedient purchasing. ${ }^{[7,8]}$ These reforms and others were expected to insure at least 3.7 million previously uninsured patients of all ages with mental illnesses, and even more with less severe mental illnesses. ${ }^{[9]}$ While mental illnesses span all age groups, the odds of having a year-long DSM-IV disorder are higher for individuals aged 19-25 than other age groups, and a majority of mental health disorders emerge by age $24 .^{[9,10]}$ The provision allowing young adults to stay as dependents on their parents' plans until their 26th birthday was one of the first provisions of the ACA to come into effect. On September 23, 2010, this provision became enforced, and since then there have been significant increases in insurance coverage for young adults aged $19-25$ years. ${ }^{[11-18]}$

While this age group has seen large increases in insurance coverage, there is little consensus on the overall effect of insurance expansion on ED use. Preliminary results suggest an increase in ED use, however considerable variability exists. ${ }^{[19-24]}$ Several studies have looked at specifically young adult patients, and use of ED for mental health reasons, also with mixed results. ${ }^{[25-30]}$ Other studies have shown that expansion of access to care is not shared equally among racial, ethnic, or gender groups. ${ }^{[31,32]}$ For example, one study from Stanford ${ }^{[25]}$ found a relative decrease in ED visits by female and African-American patients, whereas other studies did not report such discrepancies. As the ACA seeks to expand access to care for patients aged 19-25, it is important to assess how this expansion affects various groups and populations.

This study's aim is to clarify the effect of the ACA on use of the ED for psychiatric care among 19-25 year old patients who were targeted by the ACA. Additionally, this study sought to elucidate differences based on race.

\section{METHODS}

\section{Study design and setting}

This analysis was performed using data from the state of California, which was chosen as a model state for analysis due to its diversity, large population, and data availability for the years 2009-2011. Data was sourced from the State Emergency Department Databases (SEDDs) through the Healthcare Cost and Utilization Project for the Agency for Health Care Research and
Quality. ${ }^{[33]}$ The years of 2009-2011 were chosen to provide data pre- and post-implementation of the young adult provision of the ACA.

The data from the California SEDD includes: patient's demographic characteristics, diagnosis and procedure information, admission and discharge status, and expected payment source. ${ }^{[34]}$ This data also includes health insurance status such as Medicaid, Medicare, private insurance, self-pay, or other insurance; however it is not possible with data from SEDDs to determine if treated young adults were dependents on parental health plans, or if those individuals had their own Employee Subsidized Insurance versus non-group insurance. Furthermore, it is not possible to determine repeat visits by a patient. Race was self-identified by the patients.

\section{Selection of participants}

The California SEDD included information from all patients with visits to emergency departments in California during the timeframe of 2009 to 2011. Participants selected from this data included all patients either 19 to 25 or 27 to 31 with the primary diagnosis associated with an ICD-9 code 290.xx to 319.xx, representing psychiatric diagnoses. This approach may have under-identified the total number of possible participants because California SEDD does not rank diagnoses listed as primary or secondary. However because this under-representation should affect all groups uniformly given random sampling between groups, this was believed to be acceptable (see discussion in 'Limitations' section).

\section{Outcomes}

Our primary analysis measured rates of ED visits for patients carrying a primary diagnosis of non-childbirthrelated psychiatric illness. Any non-pregnant patient fitting our age restrictions and carrying a primary diagnosis of an ICD-9 code 290.xx to 319.xx was included in our analysis of these two outcomes. In line with previous studies that have analyzed ACA effects on this population, we measured all rates as visits per 1000 population based on US Census Bureau data. ${ }^{[22,26,35]}$ We created 1620 "cells" defined by sex, age (19-25 vs. 2731), race (White, Black, Hispanic, Asian/Pacific Islander, Native American, or other/mixed) and quarter for 2009-2011). In each cell, the numerator reflects the total admissions or visits for that specific sex-age-race-quarter group. The denominator is the California census estimate of the population subgroup that corresponds to that sexage-race-quarter make-up of the numerator. Secondary 
outcomes were measured with subgroup analysis looking at the effect of race, gender, and payment source on the primary outcome of ED visit rates.

\section{Analysis}

For our main analysis, we obtained California State level data on all ED visits made by people aged 19 to 31 from January 1, 2009 to December 31, 2011. With this data we compared, using a difference-in-differences approach, ${ }^{[12,25-28]}$ ED use for young adults aged 19 to 25 and 27 to 31 where visits carried a primary diagnosis of a mental health disorder. The difference-in-differences approach allows us to account for time-varying factors that would have led the treatment group (aged 19 to 25 years) to experience different rates of ED care compared to the control group (aged 27 to 31 years) after the young adult dependent provision was implemented. We determined that adults aged 27 to 31 years served as suitable controls as they carry similar lack of coverage and similar mental health concerns as those patients aged 19 to 25 years in the control group. ${ }^{[26]}$ We attempted to control for time varying trends by using the difference in rates of ED use in the treatment group before and after the implementation of the young adult provision, and subtracting this number by the difference in rates of ED use in the control group before and after implementation of the young adult dependent provision.

We defined the pre-ACA period as January 1, 2009 through August 31, 2010, and we defined the post-ACA period as January 1, 2011 through December 31, 2011. As other studies have done, ${ }^{[13,22]}$ we used September 1 , 2010 through December 31, 2010 as a wash out period to account for the fact that although the young adult dependent provision aspect of the ACA went into place on September 23, 2010, many plans are only renewed at the beginning of the new year. We exclude patients aged 26 years from our study, as some plans allow patients to remain on parental health plans until the end of the calendar year containing their $26^{\text {th }}$ birthday, and thus it is difficult to assign these patients to either group confidently. By gathering data from 2009, we were able to analyze and control for possible trends in mental health care use prior to implementation of the young adult provision on September 23, 2010.

In order test our primary outcome, we estimated the following linear regression model:

$Y_{\text {agt }}=\beta_{1}+\beta_{2}\left(\right.$ Enact $\left._{t}\right)+\beta_{3}\left(\right.$ Implement $\left._{t}\right)+\beta_{4}\left(\right.$ Treatment $_{a}$ $\times$ Enact $\left._{t}\right)+\beta_{5}\left(\right.$ Treatment $_{a} \times$ Implement $\left._{t}\right)+\beta_{6}$ Age $_{a}+\beta_{7}$ Race $_{z}+\beta_{8}$ Quarter $_{t}+\beta_{9}$ Gender $_{g}+\varepsilon_{\text {agtz }}$, where $Y_{\text {agtz }}$ is our outcome variable of interest for age $a$, gender $g$, race $z$, and quarter $t$. Enact, represents an indicator variable for quarter 2 and quarter 3 of 2010 , corresponding to the time period after the young adult dependent provision was enacted, but before it was implemented. Implement, represents the indicator variable for quarter 1 through quarter 4 of 2011, corresponding to the time period after the young adult dependent provision was implemented, and allowing for the $4^{\text {th }}$ quarter of 2010 to be a washout period. Quarter 4 of 2011, represents the latest period of available data. The variable Treatment ${ }_{a}$ is a dichotomous variable for membership in the 19 to 25 year-old age group, relative to the 27 to 31 year-old age group. In the non-interacted variable $\mathrm{Age}_{a}$ we include a full set of age indicators.

The interaction of Treatment ${ }_{a}$ with Enact ${ }_{t}$ captures the possible anticipatory changes between the time the law was enacted and the implementation of the law, approximately from late March to late September. The interaction of Treatment ${ }_{a}$ with Implement $t_{t}$, captures the average impact after the law was implemented by comparing ED visits in the treatment group relative to the control group after the young adult dependent provision was in effect. We also include dummy variables for year and quarter into the variable Quarter to $_{t}$ account for seasonality and any year-fixed effects that are common to either the treatment or control groups. Dummy variables for race and gender are designed to account for any effects that race and gender have on the primary outcome independent of our true variable of interest (Treatment ${ }_{a}$ $\times$ Implement $\left._{t}\right) . \varepsilon_{\text {agtz }}$ represents the difference in the regression line of best fit and the true data points used to build this model.

This same filtered data was used to assess the proportion of ED visits for each race that was covered by private insurance. The rationale for assessing coverage by private insurance relates to the implications of differences in private versus public coverage among racial groups. An indicator variable for private insurance was interacted with the interacted variable ( Treatment $_{a} \times$ Implement $_{t}$ ) to yield a model that would allow us to look at this differential effect of the young adult dependent provision on the proportion of ED visits that were paid for with private insurance in the 19 to 25 year-old group, relative to the 27 to 31 year-old group, by race. We estimated our linear regression models with robust Huber-White standard errors, and $P$ values were reported based on two-tailed t statistics.

This study was exempt from our IRB as it uses deidentified secondary data. 


\section{RESULTS}

Figure 1 reports sample means for the treatment and control groups throughout the entirety of the study period. Overall, the treatment group had lower rates of

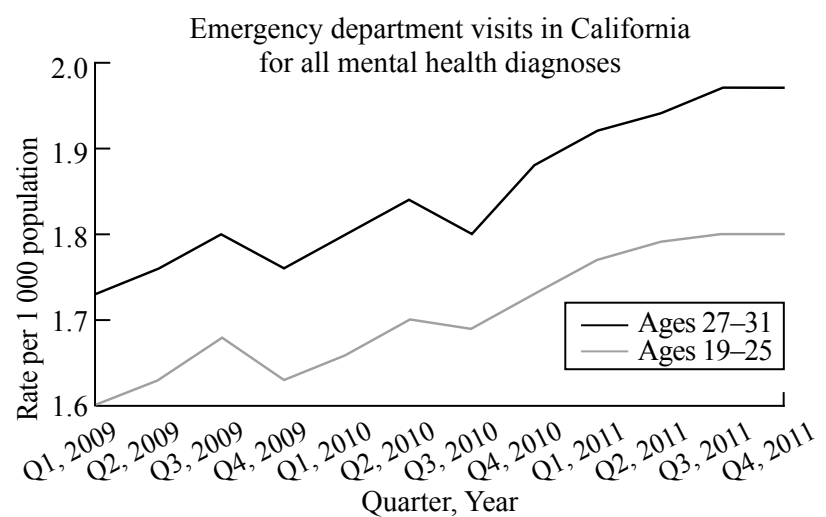

Figure 1. Quarterly emergency department visit rates, 2009-2011. *Quarterly ED visits rates. The Law was enacted Q2, 2010, and Implemented Q4, 2010.
ED utilization for psychiatric reasons compared to the control group.

\section{Main results}

Trends in outcomes, as shown in Table 1, for the treatment and control group were similar throughout the study period.

While the overall growth rate for California psychiatric ED visits rises over the extent of the three-year study period, the growth rate is significantly lower for the 19 to 25 year-old age group following the implementation of the young adult dependent provision compared to the 27 to 31 year-old age group (Table 2). Growth in ED visits was 0.05 per 1000 people $(95 \% C I-0.08$ to -0.01 ) lower among 19 to 25 year old compared to 27 to 31 year old. This differential reduction was only seen to be significantly different in the period following the implementation of the law, where the enactment period, March through September of 2010, did not have a

Table 1. California ED psychiatric visit characteristics, 2009-2011

\begin{tabular}{|c|c|c|c|c|c|c|}
\hline \multirow{2}{*}{ Characteristics } & \multicolumn{3}{|c|}{ Ages $19-25$ (ED visit per 1000 people $\left.{ }^{*}\right)$} & \multicolumn{3}{|c|}{ Ages 27-31 (ED visit per 1000 people $\left.{ }^{*}\right)$} \\
\hline & Full sample rate (SD) & Males rate (SD) & Females rate $(\mathrm{SD})$ & Full sample rate (SD) & Males rate $(\mathrm{SD})$ & Females rate $(\mathrm{SD})$ \\
\hline \multicolumn{7}{|l|}{ Race } \\
\hline All Races & $1.70(0.77)$ & $1.86(0.85)$ & $1.55(0.70)$ & $1.85(0.85)$ & $2.03(1.06)$ & $1.65(0.72)$ \\
\hline White & $1.90(0.26)$ & $1.99(0.16)$ & $1.75(0.14)$ & $1.79(0.24)$ & $1.92(0.17)$ & $1.65(0.12)$ \\
\hline Black & $2.36(0.34)$ & $2.36(0.25)$ & $2.35(0.21)$ & $2.75(0.36)$ & $2.84(0.27)$ & $2.65(0.22)$ \\
\hline Hispanic & $1.07(0.18)$ & $1.18(0.15)$ & $0.99(0.12)$ & $1.09(0.18)$ & $1.19(0.14)$ & $0.99(0.10)$ \\
\hline Asian/Pacific Islander & $0.36(0.06)$ & $0.35(0.13)$ & $0.34(0.13)$ & $0.36(0.06)$ & $0.35(0.04)$ & $0.42(0.05)$ \\
\hline Native American & $0.60(0.13)$ & $0.59(0.09)$ & $0.61(0.08)$ & $0.71(0.21)$ & $0.62(0.16)$ & $0.75(0.17)$ \\
\hline Mixed/Other & $1.73(0.27)$ & $1.95(0.21)$ & $1.52(0.19)$ & $2.03(0.31)$ & $2.25(0.23)$ & $1.82(0.16)$ \\
\hline \multicolumn{7}{|c|}{ ED visit with diagnosis $(\mathrm{dx})$ of } \\
\hline Any psychiatric $\mathrm{dx}$ & $1.70(0.77)$ & $1.86(0.85)$ & $1.55(0.70)$ & $1.85(0.85)$ & $2.03(1.06)$ & $1.65(0.72)$ \\
\hline Depression dx only ${ }^{* *}$ & $0.45(0.08)$ & $0.29(0.05)$ & $0.57(0.17)$ & $0.50(0.26)$ & $0.32(0.11)$ & $0.66(0.28)$ \\
\hline Substance use dx only & $4.22(0.50)$ & $4.87(0.89)$ & $3.61(0.40)$ & $4.42(0.55)$ & $5.21(0.94)$ & $3.67(0.43)$ \\
\hline Psychosis dx only & $0.55(0.10)$ & $0.73(0.15)$ & $0.45(0.08)$ & $0.68(0.16)$ & $0.82(0.25)$ & $0.56(0.18)$ \\
\hline Any other psychiatric $\mathrm{dx}$ & $2.8(0.34)$ & $2.51(0.60)$ & $2.98(0.70)$ & $3.13(0.39)$ & $2.81(0.64)$ & $3.39(0.79)$ \\
\hline Total ED visits & 158873 & 87195 & 71678 & 109532 & 60495 & 49037 \\
\hline
\end{tabular}

*: ED visit rates were calculated using age, sex, race and quarterly specific estimates from the US Census Bureau's state-level population estimates as the denominator. For each denominator, a specific age, sex, race and quarterly amount of ED visits made up the corresponding numerator. ${ }^{* *}$ : Any diagnosis category with "only", refers solely to that specific psychiatric diagnosis, it may include other physical diagnoses as well.

Table 2. Differential change in emergency department visits with mental health diagnoses per 1000 population for 19 to 25 year old compared to 27 to 31 year old after enactment of the Affordable Care Act dependent coverage provision*

\begin{tabular}{|c|c|c|c|c|c|c|}
\hline \multirow{2}{*}{ Outcome } & \multicolumn{3}{|c|}{ Enactment effect, 2010 (Q2-Q3) } & \multicolumn{3}{|c|}{ Implement effect, 2011 (Q1-Q4) } \\
\hline & Enactment effect & $95 \% C I$ & $P$ & Implementation effect & $95 \% C I$ & $P$ \\
\hline All ED visits & -0.01 & $-0.24,0.09$ & 0.36 & -0.05 & $-0.08,-0.01$ & $<0.001$ \\
\hline \multicolumn{7}{|l|}{ ED visits by race ${ }^{* *}$} \\
\hline Black & -0.03 & $0.00,0.05$ & 0.12 & -0.04 & $-0.06,-0.03$ & $<0.001$ \\
\hline Hispanic & -0.01 & $-0.02,0.03$ & 0.63 & 0.01 & $-0.01,0.03$ & 0.51 \\
\hline Asian/Pacific Islander & -0.02 & $-0.03,0.01$ & 0.20 & -0.02 & $-0.04,0.02$ & 0.20 \\
\hline Native American & 0.03 & $-0.01,0.10$ & 0.15 & -0.05 & $-0.08,-0.01$ & 0.04 \\
\hline Mixed/Other & 0.02 & $0.00,0.04$ & 0.09 & -0.03 & $-0.04,-0.01$ & $<0.001$ \\
\hline
\end{tabular}


significant differential effect on ED visits between age groups $\left(\beta_{1}=-0.01,95 \% C I-0.24\right.$ to -0.09$)$.

The significant differential reduction in ED visit rates following the implementation of the young adult dependent provision in 19 to 25 year old compared to 27 to 31 year old was not equally mirrored by all racial groups. While 19 to 25 year-old Whites $\left(\beta_{1}=-0.03\right.$; $95 \% C I-0.04$ to -0.01$)$, Blacks $\left(\beta_{1}=-0.04 ; 95 \% C I-0.06\right.$ to -0.03$)$, native Americans $\left(\beta_{1}=-0.05 ; 95 \% C I-0.08\right.$ to -0.01$)$, and mixed/other $\left(\beta_{1}=-0.03 ; 95 \% C I-0.04\right.$ to -0.01 ) racial groups saw significant declines in ED visit rates relative to their race congruent control group, Hispanics $\left(\beta_{1}=0.01 ; 95 \% C I-0.01\right.$ to 0.03$)$ and Asian/ Pacific-Islanders $\left(\beta_{1}=-0.02 ; 95 \% C I-0.04\right.$ to 0.02$)$ did not. No racial groups saw a significant differential change in ED visits rates during the enactment period.

Following the expansion of the young adult dependent provision, the proportion of ED visits covered by private insurance fell by $3.5 \%$ ( $95 \% C I-6.5 \%$ to $-1.5 \%)$. However, Table 3 reveals that following the implementation of the young adult dependent provision there was no overall differential increase in the share of psychiatric ED visits with private insurance among the 19 to 25 year old compared to the 27 to 31 year old $\left(\beta_{1}=0.02 ; 95 \% C I-0.01\right.$ to 0.05$)$. While there was no overall increase in the share of ED visits covered by private insurance, certain races did see differential increases in private insurance. 19 to 25 year-old Whites

Table 3. Differential change in likelihood that ED visits with behavioral health diagnoses for 19-25 year old compared to 27-31 year old are covered by private insurance, after implementation of dependent coverage provision ${ }^{*}$

\begin{tabular}{lclc}
\hline Outcome & $\begin{array}{c}\text { Coefficient } \\
\text { estimate }\end{array}$ & $\begin{array}{l}\text { Implement effect, 2011 } \\
(\mathrm{Q} 1-\mathrm{Q} 4)(95 \% C I)\end{array}$ & $P$ \\
\hline Full sample & & & \\
$\quad$ All ED visits & 0.02 & $-0.01,0.05$ & 0.603 \\
$\begin{array}{l}\text { ED visits by race } \\
\text { White }\end{array}$ & 0.08 & $0.05,0.11$ & $<0.001$ \\
Black & 0.05 & $0.02,0.08$ & 0.003 \\
Hispanic & 0.01 & $-0.02,0.04$ & 0.694 \\
Asian/Pacific Islander & -0.01 & $-0.04,0.03$ & 0.796 \\
Native American & 0.03 & $0.01,0.10$ & 0.04 \\
Mixed/other & 0.05 & $0.02,0.08$ & $<0.001$ \\
\hline
\end{tabular}

*: This table represents the coefficient estimates for the 19 to 25 yearold age group interacted with an indicator for the enactment period $\left(2^{\text {nd }}\right.$ and $3^{\text {rd }}$ quarters, 2010), and the implement period ( $1^{\text {st }}$ through $4^{\text {th }}$ quarters, 2011) in regression models of share of ED visits that were covered by private insurance, controlling for age, sex, quarter, and where appropriate race. Values for $\mathrm{p}$ are based on two-tailed $t$ statistics, and confidence intervals are based on robust Huber-White standard errors. ${ }^{* *}$ : Coefficient estimates by race were calculated from the master data file filtering for a specific race and then running the same regression as above without controlling for race. $\left(\beta_{1}=0.08 ; 95 \% C I 0.05\right.$ to 0.11$)$, Blacks $\left(\beta_{1}=0.05 ; 95 \% C I\right.$ 0.02 to 0.08$)$, mixed/other $\left(\beta_{1}=0.05 ; 95 \% C I 0.02\right.$ to 0.08$)$ and native Americans $\left(\beta_{1}=0.03,95 \% C I 0.01\right.$ to 0.10$)$ all saw significant differential increases in the share of their psychiatric ED visits that were paid for with private insurance compared to 27 to 31 year-olds. However, Hispanics $\left(\beta_{1}=0.01,95 \% C I-0.02\right.$ to 0.04$)$ and Asian/ Pacific-Islanders $\left(\beta_{1}=-0.01,95 \% C I-0.04\right.$ to 0.03$) \mathrm{did}$ not see a significant differential change in the share of ED visits paid for with private insurance.

\section{DISCUSSION}

Following the young adult dependent provision implementation, the rates of emergency department psychiatric visits were modestly less for ages 19 to 25 compared to ages 27 to 31 . These findings are largely consistent with other studies. ${ }^{[26,28,29]}$ While 0.05 ED fewer ED visits per 1000 people may seem modest, this reduction in visits is very significant when considering that the number of ED visits overall continues to increase on a yearly basis. It is also reasonable to postulate that this difference in number of psychiatric ED visits may also reflect a decrease in usage of the ED for other psychiatric or medical conditions which may be treated as an outpatient. Furthermore, ED visits cost far more than outpatient care, which makes this reduction even more significant. Additionally, following the implementation of this provision, young adults were less likely to be uninsured. However, these reductions in ED visit rates and the increase in the share of ED visits that were paid for by private insurance were not equally represented in all racial groups. Notably, Hispanics and Asian/Pacific-Islanders were the only racial groups from the data who did not see significant differential reductions in ED visit rates following the implementation of the young adult dependent provision. Additionally, Hispanics and Asian/Pacific-Islanders were the only racial groups who did not see significant differential increases in the share of ED visits that were paid for by private insurance following implementation of the law.

While previous studies ${ }^{[31,36]}$ have shown that Whites appear to gain more private insurance coverage than non-Whites, to the best of our knowledge no study has looked by race at the share of psychiatric ED visits covered by private insurance. We hypothesize that the differential increase in the share of ED visits covered by private insurance that was seen in Whites, Blacks, native Americans, and mixed/others, but not seen in Hispanics and Asian/Pacific Islanders could be partially explained 
from the fact that Hispanics and Asian/Pacific Islander's parents are less likely to be insured, and therefore these groups are less likely to gain insurance through this provision. Per a recent report on income, poverty, and health insurance status among these different races; Hispanics have some of the lowest median household incomes of all the racial groups, and have some of the highest rates of uninsured (compared to Whites). ${ }^{[37]}$ According to the same study, the Asian/Pacific Islander group was less likely to be insured (compared to Whites), but did have a lower rate of uninsured status compared to Hispanics $(16.8 \%$ vs. $30.1 \%$, with non-Hispanic Whites having $11.1 \%$ uninsured). A limitation of our study, and an area for future research is that we could not explore the socioeconomic status of the individuals, as well as their access to outpatient psychiatric care.

It is possible that a disparity exists that is not being addressed by the young adult dependent provision. This can be hypothesized to be due to the high likelihood these young adults in the Hispanic and Asian/Pacific Islander racial subgroups (when compared to Whites) are not able to gain access to insurance because their parents themselves do not have insurance. Furthermore, this could be due to differing enrollment rates in these racial subgroups, possibly due to poor understanding of a complicated American health system. Further research needs to be done to explore these possible causes.

It should be noted that the Hispanic and Asian/ Pacific Islander groups are two distinct groups. Among the Asian/Pacific Islander group, observed differences in utilization (compared to Whites) may be due to cultural differences such as utilization of community and spiritual leadership rather than the ED. Additionally, Asian/Pacific Islander immigrants who are small business owners may forego insurance coverage due to cost. Of note, in Table 1 it is seen that the Asian/Pacific Islander has a significantly lower (about one third) utilization of the ED for psychiatric reasons when compared to the Hispanic group, though this bodes unclear significance with regard to the young adult dependent provision.

While racial differences exist with regards to psychiatric health care utilization, our difference-indifferences approach which compares 19 to 25 yearold Hispanics to 27 to 31 year-old Hispanics, does not seek to compare these groups to each other with regards to the relative difference between racial groups; rather, we hoped to explore the difference within racial groups to assess if the young adult dependent provision was having an effect on each racial group, independently of the other racial groups. We hypothesize that these same racial groups that saw a reduction in the rate of ED visits (Whites, Blacks, Mixed/Other, and Native Americans), and also saw an increase in the share of their psychiatric ED visits covered by private insurance, were more likely to utilize outpatient care more effectively or efficiently due to their new access to insurance coverage.

Our study could not explore whether individuals were utilizing outpatient care or not. This is a major limitation of nearly every study that attempts to explore the effects of this young adult dependent provision, and this is a limitation that must be addressed. As we enter the era of electronic medical records, and large sweeping changes to the health care system take place, like the ACA, we need a database that can track patients' health care utilization patterns. Not just ED and inpatient, but outpatient care as well.

Of course, with respect to the recent election of Donald Trump, coverage extended under the studied dependent coverage provision may evolve. At this time it remains unclear if, when, and which, provisions of the ACA may be repealed and/or replaced. It is the belief of the authors that the dependent coverage provision extended important care to a vulnerable age group which previously had significantly higher uninsured rates, and it is the hope of the authors that this provision remains in effect after the change in administration. Should the new administration choose to revoke the dependent provision, this would likely lead to significant increases in use of the ED for psychiatric care that may be addressed more cheaply and effectively as an outpatient. This would add burden to an already stressed catch-net emergency system and would likely increase overall healthcare costs, keeping in mind that emergency medical services are widely understood to cost significantly more than outpatient medical services. Should the incoming administration enact revisions to the ACA, another potential area for improvement may be in providing additional funding for outpatient and community psychiatric resources, which may reduce the use of ED resources for potentially outpatient conditions.

Our study explored a potential health care disparity in the earliest phases of the ACA. As the ACA continues to be implemented and data becomes available, we hope this study will highlight the importance of evaluating the ACA and its effect on differing races. Our research shows the mixed effects insurance coverage can have on psychiatric health care utilization, and we hope this study serves as a catalyst for further exploration into this important topic as the ACA expands. 


\section{Limitations}

There are some limitations to our study that must be addressed. While the California SEDD's include any diagnosis the doctor entered for the patient, these diagnoses are not ranked. We, therefore, cannot ascertain from the data the primary diagnosis from a secondary or tertiary diagnosis. While one previous study ${ }^{[34]}$ suggested that the first listed diagnosis may not always represent the primary reason for an ED visit, and another previous study on ED use for psychiatric reasons has chosen to include patients' visits that had any psychiatric diagnosis provided, ${ }^{[26]}$ we chose to conservatively pick only patients who had a psychiatric diagnosis in the first diagnosis group column, out of the 25 possible diagnoses that could be given. Given that less than $5 \%$ of patients had more than 6 diagnoses listed, this did not represent a drastic change from how the previously mentioned study sampled their data. While our sampling method could bias towards under-representing the amount of psychiatric ED visits in total, as long as it randomly samples between both age groups with no clear bias, which was tested and confirmed, then the difference-in-differences approach would still be just as representative of the data as a whole. Both methods ultimately yielded similar results; however, we thought it would be prudent to analyze the data in a way that could balance the potentially existing overrepresentation of psychiatric ED visits.

\section{Funding: None.}

Ethical approval: This study was exempt from our IRB as it uses de-identified secondary data.

Conflicts of interest: No any benefits have been received from a commercial party related directly or indirectly to the study.

Contributors: Yanuck J proposed the study and wrote the first draft. All authors read and approved the final version of the paper.

\section{REFERENCES}

1 DeNavas-Walt C, Proctor BD, Smith JC. Income, poverty, and health insurance coverage in the United States: 2010; 2011. Washington.

2 Health Care Financing Administration. The Emergency Medical Treatment and Active Labor Act, as established under the Consolidated Omnibus Budget Reconciliation Act (COBRA) of 1985 (42 USC 1395 dd). Federal Register. 1994;59:32086-127.

3 Fields WW, Asplin BR, Larkin GL, Marco CA, Johnson LA, Yeh C, et al. The Emergency Medical Treatment and Labor Act as a federal health care safety net program. Acad Emerg Med. 2001;8(11):1064-9.

4 Avalere. US Census Bureau:national and state population estimates forcommunity hospitals. http://www.census.gov/ popest/data/state/totals/2011/index.html; 2011. [Accessed July
2011, 2011].

5 Lee MH, Schuur JD, Zink BJ. Owning the cost of emergency medicine: beyond 2\%. Ann Emerg Med 2013;62(5):498-505. e493.

6 Downey LVA, Zun LS, Gonzales SJ. Utilization of emergency department by psychiatric patients. Prim Psychiatry. 2009;16:60 4.

7 Busch SH, Meara E, Huskamp HA, Barry CL. Characteristics of adults with substance use disorders expected to be eligible for Medicaid under the ACA. Psychiatr Serv. 2013;64(6) 520-6.

8 Garfield RL, Zuvekas SH, Lave JR, Donohue JM. The impact of national health care reform on adults with severe mental disorders. Am J Psychiatry. 2011;168(5):486-94.

9 Kessler RC, Chiu WT, Demler O, Merikangas KR, Walters EE. Prevalence, severity, and comorbidity of 12-month DSMIV disorders in the National Comorbidity Survey Replication [published correction appears in Arch Gen Psychiatry. 2005;62(6):709]. Arch Gen Psychiatry.2005;62(6):617-27.

10 Kessler RC, Chiu WT, Colpe L, Demler O, Merikangas KR, Walters EE, et al. The prevalence and correlates of serious mental illness (SMI) in the National Comorbidity Survey Replication (NCS-R). In: Manderscheid RW, Berry JT, eds. Mental Health, United States, 2004. Rockville, MD: Substance Abuse and Mental Health Services Administration; 2006:134-48.

11 Sommers BD, Kronick R. The Affordable Care Act and insurance coverage for young adults. JAMA. 2012;307(9):9134.

12 Sommers BD, Buchmueller T, Decker SL, Carey C, Kronick $\mathrm{R}$. The Affordable Care Act has led to significant gains in health insurance and access to care for young adults. Health Aff (Millwood). 2013;32(1):165-74.

13 Mulcahy A, Harris K, Finegold K, Kellermann A, Edelman L, Sommers BD. Insurance coverage of emergency care for young adults under health reform. N Engl J Med. 2013;368(22):210512.

14 Chua KP, Sommers BD. Changes in health and medical spending among young adults under health reform. JAMA. 2014;311(23):2437-9.

15 Blumenthal D, Collins SR. Health care coverage under the Affordable Care Act-a progress report. N Engl J Med. 2014;371(3):275-81.

16 Sommers BD. Number of young adults gaining insurance due to the Affordable Care Act now tops 3 million. Washington, DC: Assistant Secretary for Planning and Education, Department of Health and Human Services, June 2012 (http://aspe.hhs.gov/ aspe/gaininginsurance/rb.shtml).

17 Collins SR, Blumenthal D, Garber T. New U.S. Census data on the uninsured underscores need for expanded coverage. New York: The Commonwealth Fund Blog, September 2013 (http:// www.commonwealthfund.org/publications/blog/2013/sep/newus-census-data-on-the-uninsured).

18 Collins SR, Rasmussen PW, Garber T, Doty MM. Covering young adults under the Affordable Care Act: the importance of outreach and Medicaid expansion. New York: The Commonwealth Fund, August 2013 (http://www.commonwealthfund. org/ /media/Files/Publications/Issue\%20Brief/2013/Aug/1701 Collins covering_young_adults_tracking_brief_final_v4.pdf).

19 Finkelstein A, Taubman S, Wright B, Bernstein M, Gruber J, Newhouse JP, et al. The Oregon health insurance experiment: evidence from the first year. Q J Econ. 2012;127(3):1057-1106. 
20 Card D, Dobkin C, Maestas N. The impact of nearly universal insurance coverage on health care utilization: evidence from medicare. Am Econ Rev. 2008;98(5):2242-58.

21 Kolstad JT, Kowalski AE. The Impact of Health Care Reform on Hospital and Preventive Care: Evidence from Massachusetts $\left({ }^{*}\right)$. J Public Econ. 2012;96(11-12):909-29.

22 Smulowitz P, Landon BE, Burke L, Baugh C, Gunn H, Lipton R. Emergency department use by the uninsured after health care reform in Massachusetts. Intern Emerg Med. 2009;4(6):501-6.

23 Anderson M, Dobkin C, Gross T. The effect of health insurance coverage on the use of medical services. American Economic Journal: Economic Policy. 2012;4(1): 1-27.

24 Anderson ML, Dobkin C, Gross T. The effect of health insurance on emergency department visits: evidence from an age-based eligibility threshold. The Review of Economics and Statistics. 2014; 96(1):189-95.

25 Hernandez-Boussard T, Burns CS, Wang NE, Baker LC, Goldstein BA. The affordable care act reduces emergency department use by young adults: evidence from three States. Health Aff (Millwood). 2014;33(9):1648-54.

26 Golberstein E, Busch SH, Zaha R, Greenfield SF, Beardslee WR, Meara E. Effect of the Affordable Care Act's young adult insurance expansions on hospital-based mental health care. Am J Psychiatry. 2015; 172(2):182-9.

27 Saloner B, Lê Cook B. An ACA provision increased treatment for young adults with possible mental illnesses relative to comparison group. Health Aff (Millwood). 2014;33(8):1425-34.

28 Antwi YA, Moriya AS, Simon K. Effects of Federal Policy to Insure Young Adults: Evidence from the 2010 Affordable Care Act Dependent Coverage Mandate. American Economic Journal: Economic Policy. 2016;(5)4: 1-28.

29 Antwi YA, Moriya AS, Simon K. Access to Health Insurance and the Use of Inpatient Medical Care: Evidence from the Affordable
Care Act Young Adult Mandate. Working paper, Indiana University. 2014.

30 Barbaresco S, Courtemanche C, Qi Y. Impacts of the Affordable Care Act Dependent Coverage Provision on Health-Related Outcomes of Young Adults. George State University. 2014.

31 O'Hara B, Brault MW. The disparate impact of the ACAdependent expansion across population subgroups. Health Serv Res. 2013;48(5):1581-92.

32 Sommers BD, Kronick R. The Affordable Care Act and insurance coverage for young adults. JAMA. 2012;307(9):913-4.

33 Agency for Healthcare Research and Quality, Healthcare Cost and Utilization Project. Overview of the State Emergency Department Databases (SEDD) [Internet]. Rockville (MD): AHRQ; Available from:http://www.hcup-us.ahrq.gov/ seddoverview.jsp

34 Senathirajah M. Special study on the meaning of the first-listed diagnosis on emergency department and ambulatory surgery records. HCUP Methods Series. Rockville, MD, Agency for Healthcare Research \& Quality. 2014.

35 Meara E, Golberstein E, Zaha R, Greenfield SF, Beardslee WR, Busch SH. Use of hospital-based services among young adults with behavioral health diagnoses before and after health insurance expansions.JAMA Psychiatry. 2014;71(4):404-11.

36 Sommers BD, Buchmueller T, Decker SL, Carey C, Kronick R. The Affordable Care Act has led to significant gains in health insurance and access to care for young adults. Health Aff (Millwood). 2013;32(1):165-74.

37 Carmen D, Proctor BD, Smith JC. Current Population Reports: Income, Poverty, and Health Insurance Coverage in the United States: 2011. 60-243.

Received November 20, 2016 Accepted after revision May 6, 2017 